\title{
Evaluation of semi-supervised learning using sparse labeling to segment cell nuclei
}

Roman Bruch, Institute of Molecular and Cell Biology, Faculty of Biotechnology, Mannheim University of Applied Sciences, Mannheim, Germany, roman.bruch@gmx.de

Rüdiger Rudolf, Institute of Molecular and Cell Biology, Faculty of Biotechnology, Mannheim University of Applied Sciences, Mannheim, Germany, r.rudolf@hs-mannheim.de

Ralf Mikut, Institute for Automation and Applied Informatics, Karlsruhe Institute of Technology, Karlsruhe, Germany, ralf.mikut@kit.edu

Markus Reischl, Institute for Automation and Applied Informatics, Karlsruhe Institute of Technology, Karlsruhe, Ger-many, markus.reischl@kit.edu

\section{Introduction}

The analysis of microscopic images from cell cultures plays an important role in the development of drugs. The segmentation of such images is a basic step to extract the viable information on which further evaluation steps are build. Classical image processing pipelines often fail under heterogeneous conditions. In the recent years deep neuronal networks gained attention due to their great potentials in image segmentation. One main pitfall of deep learning is often seen in the amount of labeled data required for training such models. Especially for $3 \mathrm{D}$ images the process to generate such data is tedious and time consuming and thus seen as a possible reason for the lack of establishment of deep learning models for 3D data.

\section{Methods}

We present a new semi-supervised training method for image segmentation of microscopic cell recordings based on an iterative approach utilizing unlabelled data during training. First, sparsely labelled data is used to train a U-Net which is then used to segment unlabelled data. After a post-processing step new labels are extracted from the segmented image which are then used in the next training step.

\section{Results}

By labeling less than one percent of the training data, a performance of $90 \%$ compared to a full annotation with 342 nuclei can be achieved. Over subsequent iterations the method can reduce the amount of falsely segmented background.

\section{Conclusion}

The proposed method reduces the amount of labels and thus the time needed for labelling to train deep learning models for image segmentation. An annotation time of 45 minutes can be reduced to half a minute while maintaining $90 \%$ of the performance. The method will be applied to $3 \mathrm{D}$ data, where a single image stack can easily contain 2000 nuclei requiring 75 hours of annotation time. 


\title{
Rapid development of tailored micro-physiological systems using novel modular plug\& play construction kit
}

\author{
Florian Schmieder, Fraunhofer Institute for Material and Beam Technology IWS, Dresden, Germany, \\ florian.schmieder@iws.fraunhofer.de \\ Stephan Behrens, Fraunhofer Institute for Material and Beam Technology IWS, Dresden, Germany, \\ stephan.behrens@iws.fraunhofer.de \\ Katja Günther, University of Applied Sciences Dresden, Dresden, Germany, katja.guenther@htw-dresden.de \\ Frank Sonntag, Fraunhofer Institute for Material and Beam Technology IWS, Dresden, Germany, \\ frank.sonntag@iws.fraunhofer.de
}

\section{Introduction}

Micro-physiological platforms (MPS) - often referred as organ-on-chip or multi-organ-chip - have an enormous potential to strengthen research in drug development, toxicological screening, personalized medicine and disease modeling. The design of such MPS is an interdisciplinary challenge. At the beginning of development, many parameters and boundary conditions (geometries, volumes, flow rates, interfaces, and coatings) are often still unknown. The probability that the first draft will work is therefore very low. A large number of design cycles are necessary to develop a miniaturized MPS with a high functional density.

\section{Methods}

However, we have identified the key elements and designed a modular plug\&play construction kit for the development of tailored MPS. The modular system provides a large number of functional, miniaturized modules such as pneumatic driven micro-pumps, oxygenators, reservoirs, sensors and cell culture compartments whose fluidic interfaces comply with the Luer-Lock standard. This allows a quick and easy combination of modules with each other, according to the intended application.

\section{Results}

By using the modular plug\&play construction kit, the successful creation of different MPS setups has achieved in the shortest time and with minimal effort. As an example, we show tailored Kidney-MPS with perfused tubular or glomerular barrier. A quick and easy integration of microfluidic components, pumps and sensors from third party suppliers is possible.

\section{Conclusion}

Thus, with the help of the modular plug\&play construction kit, a drastically reduction of financial and time expenditure of an MPS development is possible. Once an application-specific design has been successfully determined, it is possible to derive a compact MPS by following certain design rules. 


\section{High-Current Neutral Electrodes}

Norbert H. Nessler, Nessler Medizin Elektronik GmbH, Innsbruck / AUSTRIA

norbert@nessler.com

\section{Introduction}

In electrosurgery tissue is cut and coagulated by means of electrical current. The surgeon's active electrode takes the current to the site of operation from where it needs to be returned via a return electrode - the neutral electrode (NE) - to the electrosurgical generator. During this surgical process, the heating of the skin under the NE must not exceed $6^{\circ} \mathrm{C}$ according to Standard IEC 60601-2-2, $6^{\text {th }}$ Ed, 2016.

Modern surgical techniques (ablation) use higher energies with high currents and long-activation times, which increases the load (heating) on the neutral electrode.

\section{Conventional NE versus high-current NE}

For conventional NEs the Standard stipulates energy limits (heating factor $30 \mathrm{~A}^{2} \mathrm{~s}$ ) and measurement procedures for quality testing $(700 \mathrm{~mA}$ during $60 \mathrm{~s})$. For surgery with high-current, however, the only parameter stipulated in the Standard so far is a heating factor $>30 \mathrm{~A}^{2} \mathrm{~s}$. And there is no upper limit nor any recommendation for testing. The definition for high-current as ,,maximum output current" leaves room for misinterpretations, since this "maximum output current" is neither displayed anywhere nor is it readily measurable. Instead of the "maximum output current" the characteristic features for high-current NEs should be the "activation time" that is foreseeable for the scheduled surgery.

\section{Suitability assessment of high-current NEs}

Testing of NEs of adequate area (active surface $\sim 250 \mathrm{~cm}^{2}$ ) as to their load capacity in high-current mode by means of an electronic test device yielded the following results for the allowable activation times and respective load currents: $5 \mathrm{~min}$ with $1 \mathrm{~A}, 15 \mathrm{~min}$ with $700 \mathrm{~mA}$ and 2 times higher load currents if 2 NEs are used in parallel. The results also showed that testing at $1,4 \mathrm{~A}$ during $60 \mathrm{~s}$ (heating factor $120 \mathrm{~A}^{2} \mathrm{~s}$ ) could constitute a quality test for high-current NEs.

\section{Conclusion}

This paper aims at finding a workable differentiation between conventional NEs and high-current NEs and suggesting a recommendation for quality testing. 


\section{Parallel online monitoring of substance transport processes in a micro-physiological tubular barrier}

Felix Gottlöber, Fraunhofer Institute for Material and Beam Technology IWS, Dresden, Germany, felix.gottloeber@iws.fraunhofer.de Jan Sradnick, Dept. of Internal Medicine III, University Hospital Carl Gustav Carus, Dresden, Germany jan.sradnick@uniklinikum-dresden.de

Frank Sonntag, Fraunhofer Institute for Material and Beam Technology IWS, Dresden, Germany, frank.sonntag@iws.fraunhofer.de

Florian Schmieder, Fraunhofer Institute for Material and Beam Technology IWS, Dresden, Germany, florian.schmieder@iws.fraunhofer.de

\section{Introduction}

Animal experiments are, despite their disadvantages, like high costs, and their poor predictive value, still the state of the art for pharmaceutical substance testing. One reason for this problem is the incapacity of modern in vitro models, like micro-physiological systems, to perform parallel online-monitoring of transport processes over membrane barriers, such as the kidney tubular barrier. Nevertheless, the investigation of those transport processes proves to be crucial for artificial organ models. Thus the aim of this work is to develop and evaluate a compact setup for the parallel quantification of substance transport above artificial cellular membranes.

\section{Methods}

The tubular barrier was created by seeding a monolayer of immortalized human renal proximal tubule epithelial cells (RPTEC/TERT1) on 24-well transwell cell culture inserts (ThinCert ${ }^{\text {TM }}$ ). Rhodamine 123 was chosen as substrate for transport assays, due to its fluorescence and biochemical characteristics. The fluorescence measurements were performed by a miniaturized fluorescence measurement device (Fluo Sens Integrated). Two sets of LEDs and detectors were used for simultaneous measurement of fluorescence signals with excitation and emission wavelengths at $\lambda_{\mathrm{ex}}=470 / 625 \mathrm{~nm}$ and $\lambda_{\mathrm{em}}=520 / 680 \mathrm{~nm}$.

\section{Results}

The setup used for online-monitoring of Rhodamine 123 transport comprises a miniaturized fluorescence measurement device in a sealed housing suitable for handling in cell culture incubators. Reliable, parallel detection of Rhodamine 123 and fluorescence labeled human serum albumin was confirmed by comparison with an established fluorescence detection method.

\section{Conclusion}

Here, we present a setup which allows parallel online measurement of two substrates during transport assays over a micro-physiological tubular barrier. This could be a useful tool to investigate the reabsorption and secretion capability of artificial, cell-based model systems for both, glomerular as well as tubular barriers. 


\section{$\mathrm{pH}-$ and oxygen sensors based on fluorescent nanoparticles for Lab-on-Chip applications}

Alexander Netaev, Fraunhofer Institute for Microelectronic Circuits and Systems (IMS), Duisburg, Germany, alexander.netaev@ims.fraunhofer.de

Birvan Dogan Karaduman, Fraunhofer IMS, Duisburg, Germany, birvan.dogan.karaduman@ims.fraunhofer.de

Mohammed Ali Sheikh, Fraunhofer IMS, Duisburg, Germany, mohammed.ali.sheikh@ims.fraunhofer.de Nicolas Schierbaum, Fraunhofer IMS, Duisburg, Germany, nicolas.schierbaum@ims.fraunhofer.de Karsten Seidl, Department of Electronic Components and Circuits, University of Duisburg-Essen, Duisburg, Germany, karsten.seidl@uni-due.de

\section{Introduction}

We present the synthesis of silica $\left(\mathrm{SiO}_{2}\right)$-nanoparticles functionalized with fluorescent dyes. These nanoparticles were developed for multi-sensor spots directly inkjet-printed on microfluidic sytems such as Lab-onChip (LoC). The nanoparticle-based sensor spots have well-defined fluorescent properties and can be modified for sensing different environmental parameters. This was demonstrated by developing fluorescent nanoparticles whose fluorescent intensity strongly depends on the $\mathrm{pH}$-value of the solution.

\section{Methods}

We used the Stöber method (sol-gel process) to synthezise $\mathrm{SiO}_{2}$-nanoparticles. The nanoparticles were functionalized by either a $\mathrm{pH}$-sensitive (Fluorescein isothiocyanate (FITC)) or a dissolved oxygen (DO)sensitive (Tris(2,2-bipyridyl)dichlororuthenium(II)hexahydrate $\left.\left(\left[\mathrm{Ru}(\mathrm{bpy})_{3}\right]^{2+}\right)\right)$ dye. The distribution of nanoparticle size was evaluated by scanning electron microscope (SEM) and dynamic light scattering. The fluorescenct properties (excitation and emission spectrum) of nanoparticles and the $\mathrm{pH} /$ oxygen-dependency of the fluorescent intensity were measured using a microplate reader.

\section{Results}

We obtained spherical $\mathrm{SiO}_{2}$ nanoparticles with a high monodispersity (typical geometric standard deviation of 1.1). Using the Stöber process it is possible to synthesize $\mathrm{SiO}_{2}$ nanoparticles with adjustable diameters between $40 \mathrm{~nm}$ and $3.6 \mu \mathrm{m}$. Fluorescence measurements showed that the functionalization was successful with both the $\mathrm{pH}$ - and the oxygen-sensitive dye. The nanoparticles functionalized with FITC showed a linear dependence of fluorescence intensity in the $\mathrm{pH}$ range from 4.7 to 8.8 with increasing $\mathrm{pH}$ values. The relative increase in intensity was $22 \%$ per pH change relative to the maximum intensity value.

\section{Conclusion}

We successfully established a procedure for synthetizing and functionalizing $\mathrm{SiO}_{2}$-nanoparticles of defined size. The nanoparticles can be functionalized by dyes whose fluorescent properties change with altered environmental conditions such as temperature, $\mathrm{pH}, \mathrm{DO}, \mathrm{Ca}_{2}{ }^{+}$etc. Such nanoparticles are of particular interest as an ink for printing fluorescent multi-sensor spots in lab-on-chip applications. 